\title{
A HYBRID REGULARIZERS MODEL FOR MULTIPLICATIVE NOISE REMOVAL
}

\author{
Tran Thi Thu Thao \\ The University of Danang, \\ University of Economics \\ Danang, Viet Nam \\ thaotran@due.udn.vn
}

\author{
Pham Cong Thang* \\ The University of Danang, \\ University of Science and Technology \\ Danang, Viet Nam \\ pcthang@dut.udn.vn
}

\author{
Vo Duc Hong \\ The University of Danang, \\ University of Science and Technology \\ Danang, Viet Nam \\ vo.duc.hong94@gmail.com
}

\author{
Vo Duc Hoang \\ The University of Danang, \\ University of Science and Technology \\ Danang, Viet Nam \\ hoangvd.it@dut.udn.vn
}

Article history:

Received 08.02.2021, Accepted 30.06.2021

\begin{abstract}
In this paper, we propose a variational method for restoring images corrupted by multiplicative noise. Computationally, we employ the alternating minimization method to solve our minimization problem. We also study the existence and uniqueness of the proposed problem. Finally, experimental results are provided to demonstrate the superiority of our proposed hybrid model and algorithm for image denoising in comparison with state-of-the-art methods.
\end{abstract}

\section{Key words}

total variation, image restoration, multiplicative noise, minimization method

\section{Introduction}

Image denoising is an important topic in digital image processing. In this field, the main task is to reconstruct a good approximation of original $u$ from observed image $f$ and to preserve local image features for accurate and effective subsequent analysis [Pham and Kopylov, 2015].

Images are corrupted by noise due to several causes including quality of transceivers, influence of light sources or environment condition [Pham and Kopylov, 2018; Pham et al., 2018]. There are many types of noise such as Gaussian noise, Poisson noise, impulse noise, mixed noise, gamma noise etc. In this paper, we focus on the multiplicative Gamma noise removal problem.
*Corresponding author

The multiplicative noise, also known as speckle noise, usually appears in imaging techniques such as the Synthetic-aperture radar (SAR), laser, ultrasound, microscope, magnetic resonance, optical coherence tomography and so on [Steidl and Teuber, 2010; Jiang et al., 2014; Huang, Ng, and Wen 2009; Jin and Yang, 2011; Bioucas and Figueiredo, 2010; Granwehr, 2007; Chyba and Marriott, 2012; Granichin, Erofeeva, and Senin, 2018]. The noise signals are produced when the wave returns from a rough surface. If there are too many information points on the scale of the optical wavelength, the waves will affect each others and cause fraud information for the capturing devices [Vassa et al., 2008].

Assuming that the original image $u=u(x), x \in \Omega \subset$ $\mathbb{R}^{2}$ is affected by the multiplicative noise $\eta$, the multiplicative noise model is defined as follows:

$$
f=u \eta
$$

where $f$ is a corrupted image, and multiplicative Gamma noise $\eta$ follows the Gamma distribution with its probability density function given by [Aubert and Aujol, 2008; Rudin, Lions, and Osher, 2003]:

$$
p(\eta)=\frac{L^{L} \eta^{L-1}}{\Gamma(\mathbb{L})} \eta^{L-1} e^{-L \eta}, \text { for } \eta \geq 0
$$

$L$ is the positive parameter and $\Gamma($.$) is the Gamma func-$ tion, the mean value of the noise $\eta$ is 1 and the variance is $\frac{1}{L}$. 
Many approaches have been considered for the multiplicative noise removal [Liu and Fan, 2016; Ullah et al., 2017; Zhao, Wang, and Ng, 2014; Dong et al., 2017]. Among of them, Total variation (TV) based approaches have achieved great success [Li, Wang, and Zhao, 2016; Li, Lou , and Zeng, 2016; Zhou et al., 2015; Yao et al., 2019; Bai, 2019; Aubert and Aujol, 2008; Dong and Zeng, 2013]. In [Aubert and Aujol, 2008], the authors proposed a multiplicative noise removal model as follows (M1 model):

$$
\begin{aligned}
& \min _{u \in S(\Omega)}\left(\lambda \int_{\Omega}|\nabla u| d x\right. \\
&\left.+\int_{\Omega}\left(\log u+\frac{f}{u}\right) d x\right),
\end{aligned}
$$

where $u$ is the original image, $f$ is the corrupted image, $x \in \Omega, S(\Omega)=\{u \in B V(\Omega), u>0\}$ is the image space, $B V(\Omega)$ is the space of functions of bounded variation, $\lambda$ is a positive parameter, the operator $|\nabla u|$ is defined later in (5).

However,the model (1) is non-convex, and it is difficult to find its global minimal solution. To avoid the drawback, authors in [Dong and Zeng, 2013] proposed a convex variational model by adding a quadratic penalty term as follows (M2 model) :

$$
\begin{aligned}
\min _{u \in S(\Omega)}\left(\lambda \int_{\Omega}|\nabla u| d x\right. & +\int_{\Omega}\left(\log u+\frac{f}{u}\right) d x \\
& \left.+\alpha \int_{\Omega}\left(\sqrt{\frac{u}{f}}-1\right)^{2}\right)
\end{aligned}
$$

where $u$ is the original image, $f$ is the corrupted image, $x \in \Omega, S(\Omega)=\{u \in B V(\Omega), u>0\}$ is the image space, $\lambda$ and $\alpha$ are positive parameters.

The mentioned models allow us to get the good image denoising results with significantly sharp edges. However, the TV based models tend to create piecewiseconstant in restored image. It leads to undesirable problem usually called the staircase effect. To overcome the staircase effect, higher-order regularization have been considered [Liu et al., 2013; Lefkimmiatis, Bourquard, and Unser, 2012; Chen et al., 2009; Chen and Wunderli, 2002; Lysaker and Tai, 2006; Liu, Yao, and Ke 2007; Li et al., 2007; Papafitsoros and Schonlieb, 2014]. Therefore, authors in [Jiang et al., 2014] proposed an adaptive model of (1) by combinating the TV norm with a secondorder regularizer as follows (M3 model):

$$
\begin{aligned}
\min _{u \in S(\Omega)}\left(\lambda \int_{\Omega}|\nabla u| d x+\right. & \gamma \int_{\Omega}\left|\nabla^{2} u\right| d x \\
& \left.+\int_{\Omega}\left(\log u+\frac{f}{u}\right) d x\right)
\end{aligned}
$$

where $x \in \Omega, S(\Omega)=\left\{u \in B V(\Omega) \cap B V^{2}(\Omega), u>0\right\}$ is the image space, $\lambda$ and $\gamma$ are positive parameters, the operator $\left|\nabla^{2} u\right|$ is defined later in (6).
Inspired of the above studies, we propose a hybrid total variational minimization model to solve the multiplicative noise removal problem. We modify the model (2) by adding a high-order functional into the objective function and investigate an adaptive model as follows:

$$
\begin{array}{r}
\min _{u \in S(\Omega)}\left(\lambda \int_{\Omega}|\nabla u| d x+\gamma \int_{\Omega}\left|\nabla^{2} u\right| d x\right. \\
\left.+\int_{\Omega}\left(\log u+\frac{f}{u}\right) d x+\alpha \int_{\Omega}\left(\sqrt{\frac{u}{f}}-1\right)^{2}\right)
\end{array}
$$

where $x \in \Omega, S(\Omega)=\left\{u \in B V(\Omega) \cap B V^{2}(\Omega), u>0\right\}$ is the image space, $\lambda$ and $\gamma$ are positive parameters.

In this paper, our main contributions can be summarized as follows. We propose the hybrid model combining the advantages of the TV regularization and the high-order TV model. It allows to avoid the staircase effect with edge-preserving image denoising. We study the issues of existence and uniqueness of a minimizer for the proposed model. Moreover, we employ the well-known alternating minimization method to solve the minimization problem in (4). Several numerical experiments are given to show the performance of our model. In particular, a comparison with related approaches in terms of the peak signal-to-noise ratio and structural similarity index is provided as well.

The rest of the paper is organized as follows. In Section (2), we study existence and uniqueness of solution for the proposed model and present the optimization framework. Next, in Section (3), we show some numerical results of our proposed method and we compare them with the results obtained with other existing and well-known methods. Finally, some conclusions are drawn in Section (4).

\section{The Proposed Model and Method}

We can rewrite the optimization problem (4) as follows:

$$
\begin{aligned}
u^{*}=\arg \min _{u \in S(\Omega)} E(u) & \\
E(u)=\min _{u \in S(\Omega)}\left(\lambda \int_{\Omega}|\nabla u| d x\right. & +\gamma \int_{\Omega}\left|\nabla^{2} u\right| d x \\
& +\int_{\Omega}\left(\log u+\frac{f}{u}\right) d x \\
& \left.+\alpha \int_{\Omega}\left(\sqrt{\frac{u}{f}}-1\right)^{2}\right) .
\end{aligned}
$$

Definitions and notations of the spaces $B V$ and $B V^{2}$ space can be found in [Chen et al., 2009; Li et al., 2007; Chen and Wunderli, 2002; Lysaker and Tai, 2006; Liu, Yao, and Ke 2007; Papafitsoros and Schonlieb, 2014; Aubert and Kornprobst, 2006]. The discrete gradient $\nabla u$ and the second-order derivatives $\nabla^{2} u$ of an image $u$ for 
the pixel location $(i, j)$ in $u(i=1 . . M ; j=1 . . N)$ are defined as follows:

$$
\begin{gathered}
\nabla_{1} u_{i, j}=u_{i+1, j}-u_{i, j}, \quad \nabla_{2} u_{i, j}=u_{i, j+1}-u_{i, j}, \\
\nabla u_{i, j}=\left(\nabla_{1} u_{i, j}, \nabla_{2} u_{i, j}\right) \\
\left|\nabla u_{i, j}\right|=\sqrt{\left(\nabla_{1} u_{i, j}\right)^{2}+\left(\nabla_{2} u_{i, j}\right)^{2}} \\
\nabla_{11} u_{i, j}=u_{i+1, j}-2 u_{i, j}+u_{i-1, j} \\
\nabla_{22} u_{i, j}=u_{i, j+1}-2 u_{i, j}+u_{i, j-1}
\end{gathered}
$$

$$
\begin{aligned}
& \nabla_{12} u_{i, j}=u_{i, j}-u_{i, j-1}-u_{i-1, j}+u_{i-1, j-1}, \\
& \nabla_{12} u_{i, j}=\nabla_{21} u_{i, j}
\end{aligned}
$$

$\left|\nabla^{2} u\right|=$

$\sqrt{\left(\nabla_{11} u_{i, j}\right)^{2}+\left(\nabla_{12} u_{i, j}\right)^{2}+\left(\nabla_{21} u_{i, j}\right)^{2}+\left(\nabla_{22} u_{i, j}\right)^{2}}$.

Motivated by [Aubert and Aujol, 2008; Dong and Zeng, 2013], we have the following theorem to show the existence and uniqueness of the optimization solution to the problem (4). First, we show that $E(\cdot)$ is a convex functional. Second, we show that $E(\cdot)$ has a lower bound. These two facts together imply the existence and uniqueness of solution for the minimization problem (4).

Theorem 1. The optimization problem (4) has a solution. Moreover, if $\alpha \geq \frac{2 \sqrt{6}}{9}$, the solution is unique.

Proof. Let $u^{(k)}$ be a bounded minimizing sequence. By the compactness property in the space of bound variation $B V(\Omega)$ and $B V^{2}(\Omega)$, there exists $u^{*} \in B V(\Omega) \cap$ $B V^{2}(\Omega)$, such that $u^{(k)}$ converges weakly to $u^{*} \in$ $B V(\Omega) \cap B V^{2}(\Omega)$ and $u^{(k)}$ converges strongly to $u^{*}$ in $L^{1}(\Omega)$. According to [Chen and Wunderli, 2002; Papafitsoros and Schonlieb, 2014; Li et al., 2007; Dong and Zeng, 2013], we know that the total variation terms are convex, and the fidelity term in (4) are strictly convex when $\alpha \geq \frac{2 \sqrt{6}}{9}$. Therefore, if $\alpha \geq \frac{2 \sqrt{6}}{9}$, we obtain that $E(z)$ is strongly convex. According to Fatou's lemma [Feinberg, Kasyanov, and Zadoianchuk, 2014], we obtain :

$$
E(u) \geq E\left(u^{*}\right) .
$$

Thus, $u^{*}$ is a minimizer of the optimization problem (4).

There are many methods which can be employed to obtain the solution of the optimization problem (4), for instance, the primal-dual algorithm, the split-Bregman algorithm, alternating minimization method [Chambolle, 2004; Chan et al., 2011; Goldstein and Osher, 2008; Pham et al., 2019]. In this article, we solve the optimization problem (4) via the alternating direction algorithm which is a variant of the classical augmented Lagrangian multiplier method [Wu and Tai, 2010].

Following the popular alternating minimization method [Chan et al., 2011; Wang et al., 2008; Tai Hahn, and Chung, 2011], we introduce three new variables $(d, g, z)$ and rewrite (4) in the constrained discrete optimization problem as follows:

$$
\begin{aligned}
\min _{z, d, g}\left(\lambda\|d\|_{1}\right. & +\gamma\|g\|_{1}+\left\langle\mathbf{1}, \log z+\frac{f}{z}\right\rangle \\
& \left.+\alpha\left(\sqrt{\frac{z}{f}}-1\right)^{2}\right) \\
\text { s.t. } d & =\nabla u, g=\nabla^{2} u, z=u .
\end{aligned}
$$

The augmented Lagrangian functional for the constrained optimization problem (7) is defined as:

$$
\begin{aligned}
& \min _{z, d, g, \rho_{1}, \rho_{2}, \rho_{3}}\left(\lambda\|d\|_{1}+\gamma\|g\|_{1}+\left\langle\mathbf{1}, \log z+\frac{f}{z}\right\rangle\right. \\
& +\alpha\left(\sqrt{\frac{z}{f}}-1\right)^{2}-\left\langle\rho_{1}, d-\nabla u\right\rangle+\frac{\eta_{1}}{2}\|d-\nabla u\|_{2}^{2} \\
& -\left\langle\rho_{2}, g-\nabla^{2} u\right\rangle+\frac{\eta_{2}}{2}\left\|g-\nabla^{2} u\right\|_{2}^{2} \\
& \left.-\left\langle\rho_{3}, z-u\right\rangle+\frac{\eta_{3}}{2}\|z-u\|_{2}^{2}\right),
\end{aligned}
$$

where $\eta_{1}, \eta_{2}, \eta_{3}$ - positive parameters; $\rho_{1}, \rho_{2}, \rho_{3}$ - with Lagrangian multipliers.

The minimization method to solve the optimization problem (8) can be expressed as follows:

$$
\begin{aligned}
& u^{(k+1)}=\underset{u}{\arg \min }\left(-\left\langle\rho_{1}, d-\nabla u\right\rangle+\frac{\eta_{1}}{2}\|d-\nabla u\|_{2}^{2}\right. \\
& -\left\langle\rho_{2}, g-\nabla^{2} u\right\rangle+\frac{\eta_{2}}{2}\left\|g-\nabla^{2} u\right\|_{2}^{2} \\
& \left.-\left\langle\rho_{3}, z-u\right\rangle+\frac{\eta_{3}}{2}\|z-u\|_{2}^{2}\right), \\
& d^{(k+1)}=\underset{d}{\arg \min }\left(\lambda\|d\|_{1}-\left\langle\rho_{1}, d-\nabla u\right\rangle\right. \\
& \left.+\frac{\eta_{1}}{2}\|d-\nabla u\|_{2}^{2}\right), \\
& g^{(k+1)}=\underset{g}{\arg \min }\left(\gamma\|g\|_{1}-\left\langle\rho_{2}^{(k)}, g-\nabla^{2} u^{(k+1)}\right\rangle\right. \\
& \left.+\frac{\eta_{2}}{2}\left\|g-\nabla^{2} u^{(k+1)}\right\|_{2}^{2}\right), \\
& z^{(k+1)}=\underset{z}{\arg \min }\left(\left\langle\mathbf{1}, \log z+\frac{f}{z}\right\rangle+\alpha\left(\sqrt{\frac{z}{f}}-1\right)^{2}\right. \\
& \left.-\left\langle\rho_{3}^{(k)}, z-u^{(k+1)}\right\rangle+\frac{\eta_{3}}{2}\left\|z-u^{(k+1)}\right\|_{2}^{2}\right),
\end{aligned}
$$

with update for $\rho_{1}^{(k+1)}, \rho_{2}^{(k+1)}, \rho_{3}^{(k+1)}$ :

$$
\left\{\begin{array}{l}
\rho_{1}^{(k+1)}=\rho_{1}^{(k)}+\eta_{1}\left(\nabla u^{(k+1)}-d^{(k+1)}\right), \\
\rho_{2}^{(k+1)}=\rho_{2}^{(k)}+\eta_{2}\left(\nabla^{2} u^{(k+1)}-g^{(k+1)}\right), \\
\rho_{3}^{(k+1)}=\rho_{3}^{(k)}+\eta_{3}\left(u^{(k+1)}-z^{(k+1)}\right) .
\end{array}\right.
$$


The $u$ subproblem is given by:

$u^{(k+1)}=\underset{u}{\arg \min }\left(-\left\langle\rho_{1}, d-\nabla u\right\rangle+\frac{\eta_{1}}{2}\|d-\nabla u\|_{2}^{2}\right.$

$-\left\langle\rho_{2}, g-\nabla^{2} u\right\rangle+\frac{\eta_{2}}{2}\left\|g-\nabla^{2} u\right\|_{2}^{2}$

$\left.-\left\langle\rho_{3}, z-u\right\rangle+\frac{\eta_{3}}{2}\|z-u\|_{2}^{2}\right)$

$=\frac{\eta_{1}}{2}\left\|d-\nabla u^{(k+1)}-\frac{\rho_{1}^{(k)}}{\eta_{1}}\right\|_{2}^{2}$

$+\frac{\eta_{2}}{2}\left\|g-\nabla^{2} u^{(k+1)}-\frac{\rho_{2}^{(k)}}{\eta_{2}}\right\|_{2}^{2}+\frac{\eta_{3}}{2}\left\|z-u-\frac{\rho_{3}^{(k)}}{\eta_{3}}\right\|_{2}^{2}$

Thus, we get:

$\eta_{1} \nabla^{T}\left(\nabla u+\frac{\rho_{1}^{(k)}}{\eta_{1}}-d^{(k)}\right)+\eta_{2} \nabla^{2^{T}}\left(\nabla^{2} u+\frac{\rho_{2}^{(k)}}{\eta_{2}}-g^{(k)}\right)$

$+\eta_{3}\left(u+\frac{\rho_{3}^{(k)}}{\eta_{3}}-z^{(k)}\right)=0$.

We can rewrite the equation as follows:

$$
\begin{aligned}
& \left(\eta_{1} \nabla^{T} \nabla+\eta_{2} \nabla^{2^{T}} \nabla^{2}+\eta_{3}\right) u^{(k+1)} \\
& =\eta_{1} \nabla^{T}\left(d^{(k)}-\frac{\rho_{1}^{(k)}}{\eta_{1}}\right)+\eta_{2} \nabla^{2^{T}}\left(g^{(k)}-\frac{\rho_{2}^{(k)}}{\eta_{2}}\right) \\
& +\eta_{3}\left(z^{(k)}-\frac{\rho_{3}^{(k)}}{\eta_{3}}\right) .
\end{aligned}
$$

It is obvious that system (10) is linear and symmetric positive definite, therefore $z^{(k+1)}$ can be efficiently solved by fast Fourier transform (FFT) [Wang et al., 2008; Pham, Tran, and Gamard, 2020], under the periodic boundary conditions:

$$
u^{(k+1)}=\mathcal{F}^{-1}\left(\frac{\mathcal{F}(\mathcal{G})}{\eta_{1} \mathcal{F}\left(\nabla^{T} \nabla\right)+\eta_{2} \mathcal{F}\left(\nabla^{2^{T}} \nabla^{2}\right)+\eta_{3}}\right)
$$

where $\mathcal{F}$ and $\mathcal{F}^{-1}$ are the forward and inverse Fourier transform operators, and

$$
\begin{aligned}
\mathcal{G}= & \left(\eta_{1} \nabla^{T}\left(d^{(k)}-\frac{\rho_{1}^{(k)}}{\eta_{1}}\right)+\eta_{2} \nabla^{2^{T}}\left(g^{(k)}-\frac{\rho_{2}^{(k)}}{\eta_{2}}\right)\right. \\
& \left.+\eta_{3}\left(z^{(k)}-\frac{\rho_{3}^{(k)}}{\eta_{3}}\right)\right)
\end{aligned}
$$

The $d$ and $g$ subproblems are given by:

$$
\begin{aligned}
d^{(k+1)}= & \underset{d}{\arg \min }\left(\lambda\|d\|_{1}-\left\langle\rho_{1}, d-\nabla u\right\rangle\right. \\
& \left.+\frac{\eta_{1}}{2}\|d-\nabla u\|_{2}^{2}\right) \\
& =\underset{d}{\arg \min }\left(\lambda\|d\|_{1}+\right. \\
& \left.\frac{\eta_{1}}{2}\left\|d-\nabla u^{(k+1)}-\frac{\rho_{1}^{(k)}}{\eta_{1}}\right\|_{2}^{2}\right) .
\end{aligned}
$$

$$
\begin{aligned}
g^{(k+1)}= & \underset{g}{\arg \min }\left(\gamma\|g\|_{1}-\left\langle\rho_{2}^{(k)}, g-\nabla^{2} u^{(k+1)}\right\rangle\right. \\
& \left.+\frac{\eta_{2}}{2}\left\|g-\nabla^{2} u^{(k+1)}\right\|_{2}^{2}\right) \\
& =\underset{g}{\arg \min }\left(\gamma\|g\|_{1}+\right. \\
& \left.\frac{\eta_{2}}{2}\left\|g-\nabla^{2} u^{(k+1)}-\frac{\rho_{2}^{(k)}}{\eta_{2}}\right\|_{2}^{2}\right) .
\end{aligned}
$$

Similarly to [Goldstein and Osher, 2008], generalized shrinkage formula can be employed for solving the $d$ and $g$ subproblems as follows:

$d^{(k+1)}=$

$\frac{\nabla u^{(k+1)}+\frac{\rho_{1}^{(k)}}{\eta_{1}}}{\left|\nabla u^{(k+1)}+\frac{\rho_{1}^{(k)}}{\eta_{1}}\right|} \cdot \max \left(\left|\nabla u^{(k+1)}+\frac{\rho_{1}^{(k)}}{\eta_{1}}\right|-\frac{\lambda}{\eta_{1}}, 0\right)$,

$g^{(k+1)}=$

$\frac{\nabla^{2} u^{(k+1)}+\frac{\rho_{2}^{(k)}}{\eta_{2}}}{\left|\nabla^{2} u^{(k+1)}+\frac{\rho_{2}^{(k)}}{\eta_{2}}\right|} \cdot \max \left(\left|\nabla^{2} u^{(k+1)}+\frac{\rho_{2}^{(k)}}{\eta_{2}}\right|-\frac{\gamma}{\eta_{2}}, 0\right)$.

The $z$ subproblem is given by:

$$
\begin{aligned}
z^{(k+1)} & =\underset{z}{\arg \min }\left(\left\langle\mathbf{1}, \log z+\frac{f}{z}\right\rangle+\alpha\left(\sqrt{\frac{z}{f}}-1\right)^{2}\right. \\
& \left.-\left\langle\rho_{3}^{(k)}, z-u^{(k+1)}\right\rangle+\frac{\eta_{3}}{2}\left\|z-u^{(k+1)}\right\|_{2}^{2}\right) \\
& =\underset{z}{\arg \min }\left(\left\langle\mathbf{1}, \log z+\frac{f}{z}\right\rangle+\alpha\left(\sqrt{\frac{z}{f}}-1\right)^{2}\right. \\
& \left.+\frac{\eta_{3}}{2}\left\|z-u^{(k+1)}-\frac{\rho_{3}^{(k)}}{\eta_{3}}\right\|_{2}^{2}\right) .
\end{aligned}
$$

Therefore, we get:

$$
\begin{aligned}
F(z) & =\left(\frac{1}{z}-\frac{f}{z^{2}}\right)+\alpha\left(\frac{1}{f}-\frac{1}{\sqrt{f z}}\right) \\
& +\eta_{3}\left(z-u^{(k+1)}\right)-\rho_{3}^{(k)}=0 .
\end{aligned}
$$

Applying the Newton's Method, we obtain:

$$
z^{(k+1)}=z^{(k)}-\frac{F\left(z^{(k)}\right)}{F^{\prime}\left(z^{(k)}\right)},
$$




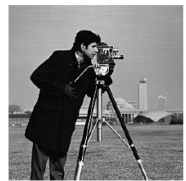

(a)

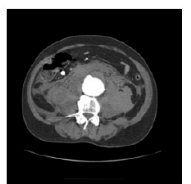

(a)

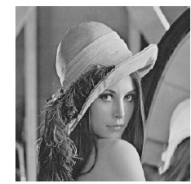

(b)

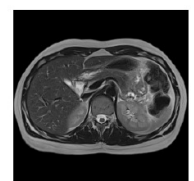

(b)

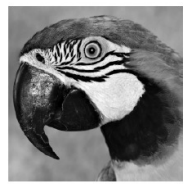

(c)

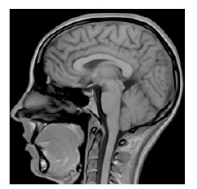

(c)

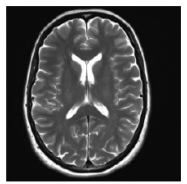

(d)

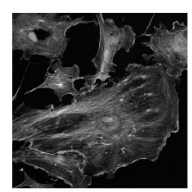

(d)
Figure 1. Test images

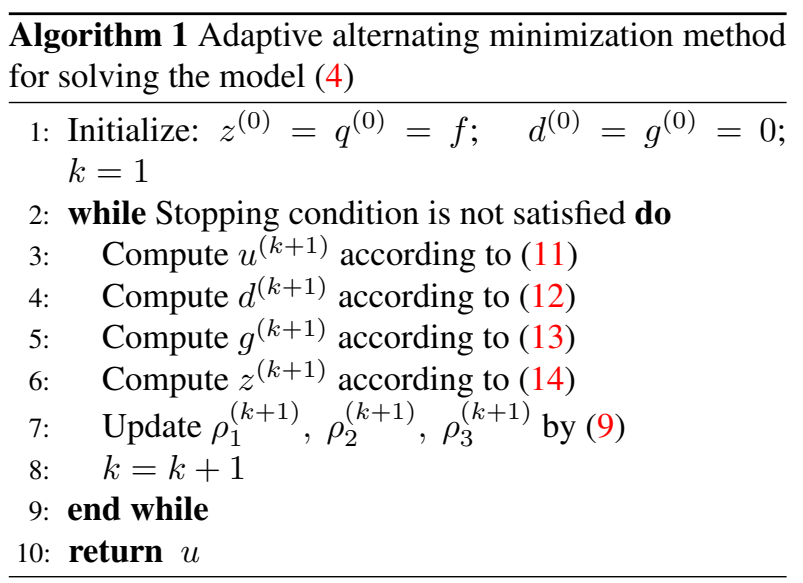

where

$$
F^{\prime}\left(z^{(k)}\right)=\left(-\frac{1}{z^{2}}+2 \frac{f}{z^{3}}\right)+\frac{\alpha}{2} \frac{1}{\sqrt{f z^{3}}}+\eta_{3} .
$$

The complete method is summarized in Algorithm (1).

We need a stopping criterion for the iteration: we end the loop if the maximum number of allowed outer iterations $N$ has been carried out (to guarantee an upper bound on running time) or the following condition is satisfied for some prescribed tolerance $\varsigma$ :

$$
\frac{\left\|u^{(k)}-u^{(k-1)}\right\|_{2}}{\left\|u^{(k)}\right\|_{2}}<\varsigma,
$$

where $\varsigma$ is a small positive parameter. For our experiments, we set tolerance in (15): $\varsigma=0.00001$ and $N=200$.

\section{Experimental Results}

In this section, we present some numerical results to illustrate the competitive performance of the proposed model for multiplicative noise removal. We compared our recovered results with those of the M1 model (1), the M2 model (2) and the M3 model (3). The compared models are implemented by the state-of-the-art alternating minimization algorithm. Empirically, all images are processed with the equivalent parameters $\eta_{1}=$
$0.01, \eta_{2}=0.01, \eta_{3}=1$ in our numerical implementation. All experiments were carried out in Windows 10 and Matlab running on a desktop equipped with an Intel Core $-\mathrm{i} 5,2.4 \mathrm{GHz}$ and $8 \mathrm{~GB}$ of RAM.

To assess quality of the restoration results, we use PSNR (Peak Signal-to-Noise Ratio), SSIM (Structural Similarity Index Measure) [Wang and Bovik, 2006] and visual quality. The test images of size $256 \times 256$ are shown in Figure (1). In our example, our images are corrupted by multiplicative gamma noise with $L=25$ and $L=10$. In Figures (2) and (4), we show the results of compared methods for noise levels $L=25$, while in Figures (3) and (5), we show the results of compared methods for noise levels $L=10$. In Figures (2)a, (3) $\mathrm{a}$, (4)a and (5)a, we represent the noisy images. In the others, Figures (2)(b)-(2)(e), (3)(b)-(3)(e),(4)(b)-(4)(e), (5)(b)-(5)(e), we show respectively the reconstructions given by compared methods.

For a better visual comparison, we have presented the zoomed details of the restored images in Figures (6), (7),(8) and (9). In these Figures, we include zoomed details of the original and noisy images in the first and second column, respectively. From the details in Figures (6), (7), (8) and (9), we can see that the our model can get better visual improvement than the others.

For quantitative performance comparison, we compare the denoised results in terms of SSIM and PSNR reported in Tables (1) and (2) for noise level $L=25$, in Tables (3) and (4) for noise level $L=10$ (the best results are highlighted in bold).

Table 1. PSNR values for noisy images and restored images with noise level $L=25$

\begin{tabular}{|c|c|c|c|c|c|}
\hline \multirow{2}{*}{ Image } & \multicolumn{5}{|c|}{ PSNR } \\
\cline { 2 - 6 } & Noisy & Model M1 & Model M2 & Model M3 & Ours \\
\hline Cameraman & 19.7802 & 25.6086 & 25.2369 & $\mathbf{2 6 . 1 3 8 2}$ & 25.9802 \\
\hline Lena & 19.5004 & 26.3679 & 26.2001 & $\mathbf{2 6 . 6 6 8 5}$ & 26.6609 \\
\hline Parrot & 20.5033 & 26.6007 & 26.4690 & 27.2063 & $\mathbf{2 7 . 3 6 8 5}$ \\
\hline Brain & 24.3012 & 28.1683 & 28.7798 & 28.3065 & $\mathbf{2 9 . 0 4 0 2}$ \\
\hline Abdomen & 24.2525 & 28.0374 & 28.1557 & 28.9483 & $\mathbf{2 9 . 5 5 4 9}$ \\
\hline Head & 22.0944 & 28.0910 & 28.5307 & 28.3362 & $\mathbf{2 8 . 8 6 3 7}$ \\
\hline CT & 26.5314 & 30.8103 & 31.0191 & 31.1879 & $\mathbf{3 1 . 9 5 5 0}$ \\
\hline Fluocells & 26.4711 & 25.9226 & 25.8966 & 26.9535 & $\mathbf{2 7 . 7 5 6 1}$ \\
\hline \hline Average & 22.9293 & 27.4509 & 27.5360 & 27.9807 & $\mathbf{2 8 . 3 8 9 9}$ \\
\hline
\end{tabular}

We can clearly see that our proposed method gets better results than other relative methods in the vast majority of cases. It again demonstrates effectiveness and efficiency of the proposed approach for suppressing multiplicative noise in terms of restoration accuracy and visual quality.

\section{Conclusions}

In this paper, we have researched the hybrid regularizers model, combining the first and second-order TV for denoising image corrupted by the multiplicative Gamma 

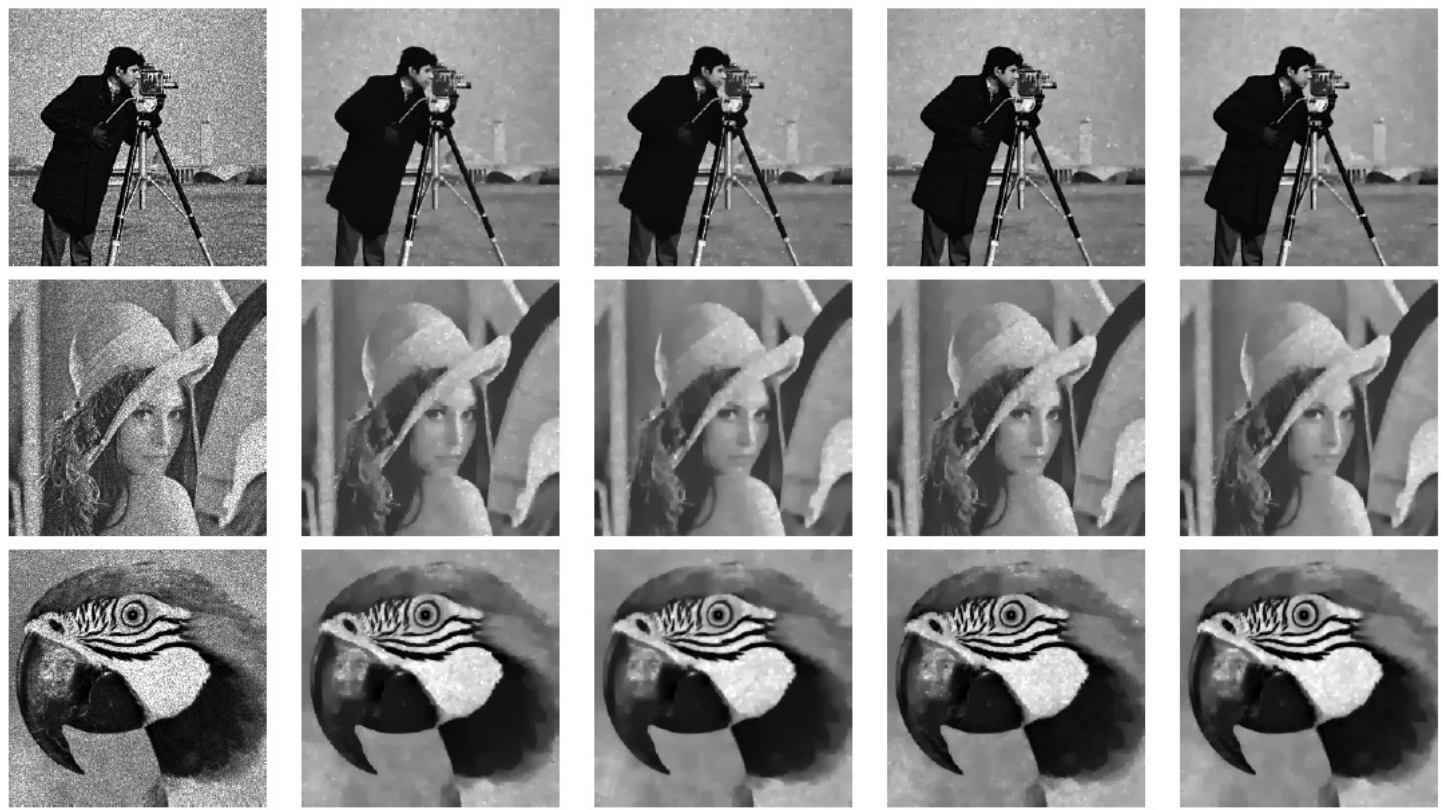

(a)

(b)

(c)



(d)

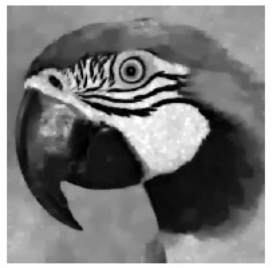

(e)

Figure 2. Recovered results for images. (a) Noisy image ( $L=25$ ), (b) M1 model, (c) M2 model, (d) M3 model, (e) Ours
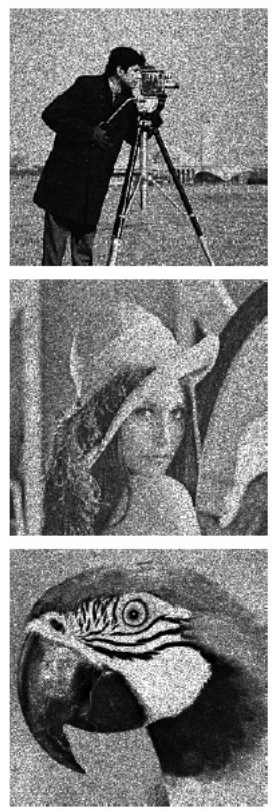

(a)


(b)
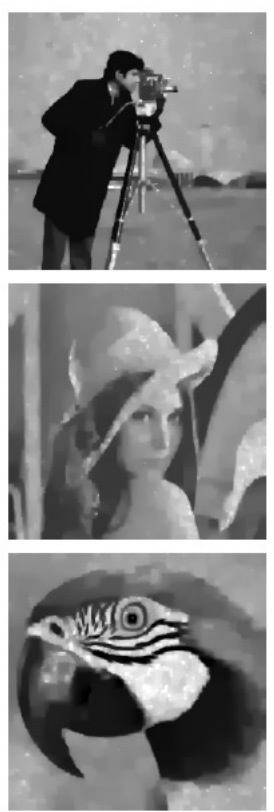

(c)
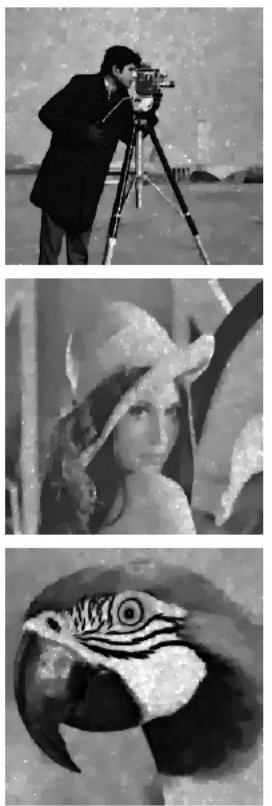

(d)
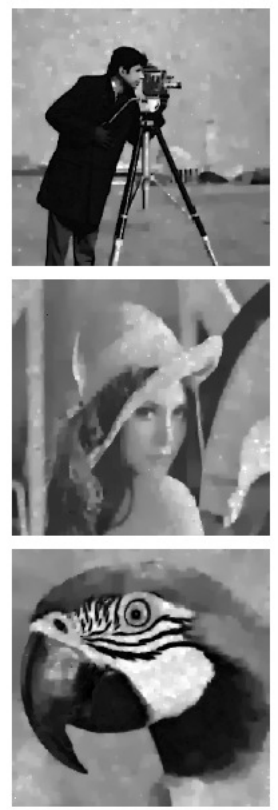

(e)

Figure 3. Recovered results for images. (a) Noisy image ( $L=10$ ), (b) M1 model, (c) M2 model, (d) M3 model, (e) Ours

Table 2. SSIM values for noisy images and restored images with noise level $L=25$

\begin{tabular}{|c|c|c|c|c|c|}
\hline \multirow{2}{*}{ Image } & \multicolumn{5}{|c|}{ PSNR } \\
\cline { 2 - 6 } & Noisy & Model M1 & Model M2 & Model M3 & Ours \\
\hline Cameraman & 0.49846 & 0.7408 & 0.7551 & 0.7642 & $\mathbf{0 . 7 7 4 1}$ \\
\hline Lena & 0.42893 & 0.7481 & 0.7528 & 0.7611 & $\mathbf{0 . 7 6 9 6}$ \\
\hline Parrot & 0.53286 & 0.7960 & 0.8007 & 0.8150 & $\mathbf{0 . 8 2 7 8}$ \\
\hline Brain & 0.76224 & 0.9271 & 0.9346 & 0.9297 & $\mathbf{0 . 9 3 5 3}$ \\
\hline Abdomen & 0.80878 & 0.8162 & 0.8180 & 0.8400 & $\mathbf{0 . 8 4 9 3}$ \\
\hline Head & 0.68914 & 0.8409 & 0.8495 & 0.8426 & $\mathbf{0 . 8 5 0 3}$ \\
\hline CT & 0.83851 & 0.8329 & 0.8360 & 0.8428 & $\mathbf{0 . 8 5 3 5}$ \\
\hline Fluocells & 0.85083 & 0.6960 & 0.6897 & 0.7678 & $\mathbf{0 . 8 0 5 5}$ \\
\hline \hline Average & 0.6762 & 0.7998 & 0.8046 & 0.8204 & $\mathbf{0 . 8 3 3 2}$ \\
\hline
\end{tabular}

noise. Computationally, an improved highly efficient alternating minimization algorithm is employed for solving the proposed optimization problem. Finally, compared with the existing state-of-the-art TV based models, the experimental results demonstrate that the our proposed approach outperforms other related approaches for removing multiplicative noise both in quantitative and 

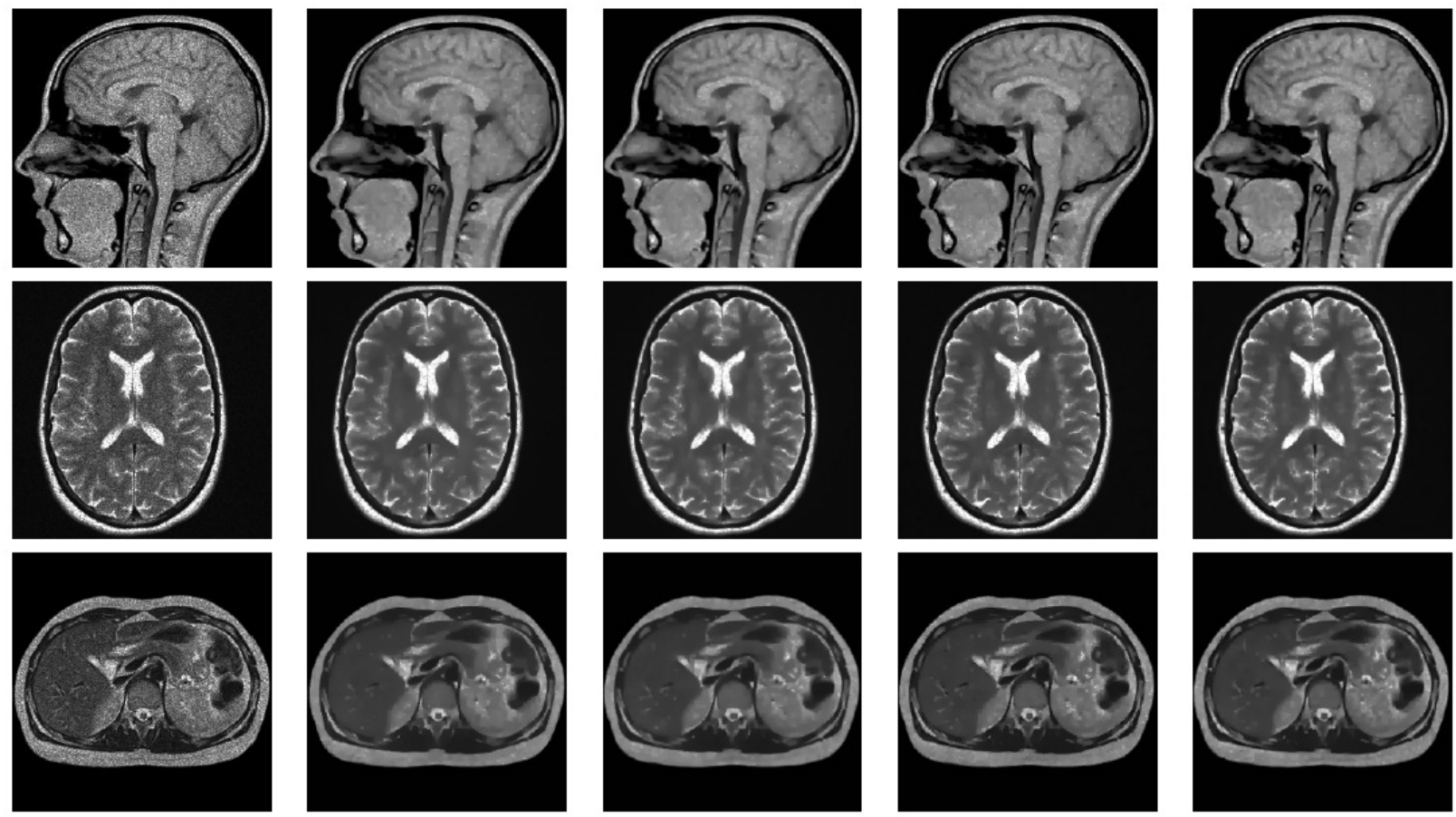

(a)

(b)

(c)

(d)

(e)

Figure 4. Recovered results for images. (a) Noisy image ( $L=25$ ), (b) M1 model, (c) M2 model, (d) M3 model, (e) Ours

Table 3. PSNR values for noisy images and restored images with noise level $L=10$

\begin{tabular}{|c|c|c|c|c|c|}
\hline \multirow{2}{*}{ Image } & \multicolumn{5}{|c|}{ PSNR } \\
\cline { 2 - 6 } & Noisy & Model M1 & Model M2 & Model M3 & Ours \\
\hline Cameraman & 16.3002 & 23.3517 & 23.1299 & $\mathbf{2 4 . 0 9 2 2}$ & 23.9874 \\
\hline Lena & 16.0233 & 24.4260 & 24.4095 & 24.7451 & $\mathbf{2 5 . 1 6 9 4}$ \\
\hline Parrot & 17.0149 & 24.3129 & 24.3015 & 24.9443 & $\mathbf{2 5 . 4 8 5 6}$ \\
\hline Brain & 20.6261 & 25.4421 & 26.0011 & 25.6761 & $\mathbf{2 6 . 6 2 6 3}$ \\
\hline Abdomen & 20.4606 & 25.6623 & 25.7900 & 26.4113 & $\mathbf{2 7 . 4 1 6 8}$ \\
\hline Head & 18.2888 & 25.5532 & 25.9455 & 25.9190 & $\mathbf{2 6 . 4 7 3 0}$ \\
\hline CT & 22.7626 & 28.3723 & 28.6713 & 28.6720 & $\mathbf{2 9 . 7 8 8 4}$ \\
\hline Fluocells & 22.5595 & 24.4637 & 24.5098 & 25.1329 & $\mathbf{2 6 . 2 2 2 9}$ \\
\hline \hline Average & 19.2545 & 25.1980 & 25.3448 & 25.6991 & $\mathbf{2 6 . 3 9 6 2}$ \\
\hline
\end{tabular}

Table 4. SSIM values for noisy images and restored images with noise level $L=10$

\begin{tabular}{|c|c|c|c|c|c|}
\hline \multirow{2}{*}{ Image } & \multicolumn{5}{|c|}{ SSIM } \\
\cline { 2 - 6 } & Noisy & Model 1 & Model 2 & Model 3 & Ours \\
\hline Cameraman & 0.40312 & 0.6972 & 0.7071 & 0.7190 & $\mathbf{0 . 7 3 5 6}$ \\
\hline Lena & 0.30407 & 0.6906 & 0.7009 & 0.7104 & $\mathbf{0 . 7 2 8 0}$ \\
\hline Parrot & 0.41961 & 0.7497 & 0.7548 & 0.7725 & $\mathbf{0 . 7 9 4 2}$ \\
\hline Brain & 0.64233 & 0.8808 & 0.8876 & 0.8857 & $\mathbf{0 . 8 8 7 3}$ \\
\hline Abdomen & 0.72162 & 0.7627 & 0.7654 & 0.8011 & $\mathbf{0 . 8 1 1 9}$ \\
\hline Head & 0.59478 & 0.7755 & 0.7902 & 0.7933 & $\mathbf{0 . 8 0 1 0}$ \\
\hline CT & 0.768 & 0.7941 & 0.7980 & 0.8106 & $\mathbf{0 . 8 1 9 6}$ \\
\hline Fluocells & 0.73204 & 0.6333 & 0.6266 & 0.7134 & $\mathbf{0 . 7 6 4 3}$ \\
\hline \hline Average & 0.5732 & 0.7480 & 0.7538 & 0.7758 & $\mathbf{0 . 7 9 2 7}$ \\
\hline
\end{tabular}

qualitative terms.

The proposed method can be appiled for multiplicative noise removal in some practical applications, e.g. Optical coherence tomography, Laser Doppler Vibration applications, etc. Optical coherence tomography (OCT) is an imaging technique that depends fundamentally on the coherence of the light used in the imaging process, and multiplicative noise is a significant issue in OCT [Liu, Zaki, and Renaud, 2018; Goodman, 2020]. In applications of Laser Doppler Vibrations, speckles noise generated by the relative in-plane motion between the Laser Doppler Vibrometry (LDV) and the target damages the quality of the LDV-captured signal severely [Tabatabai et al., 2013; Lv et al., 2019; Zhu and Baets, 2019].

\section{Acknowledgements}

The authors thank the anonymous reviewers and Editor for their insightful comments and suggestions. This research is funded by Funds for Science and Technology Development of the University of Danang under project number B2019-DN02-62.

\section{References}

Aubert, G., and Aujol, J.F. (2008). A variational approach to removing multiplicative noise. SIAM J. Appl. Math., 68 (4), pp. 925-946.

Aubert, G. and Kornprobst, P. (2006). Mathematical Problems in Image Processing: partial differential equations and the calculus of variations. Appl. Math. Sci., 147 2nd ed., Springer-Verlag New York, 379 p.

Bai, L. (2019). A new nonconvex approach for image restoration with Gamma noise. Comput. Math. Appl., 77 (10), pp. 2627-2639.

Bioucas-Dias, J., and Figueiredo, M. (2010) Multiplicative noise removal using variable splitting and constrained optimization. IEEE Trans. Image Process., 19 (7), pp. 1720-1730. 

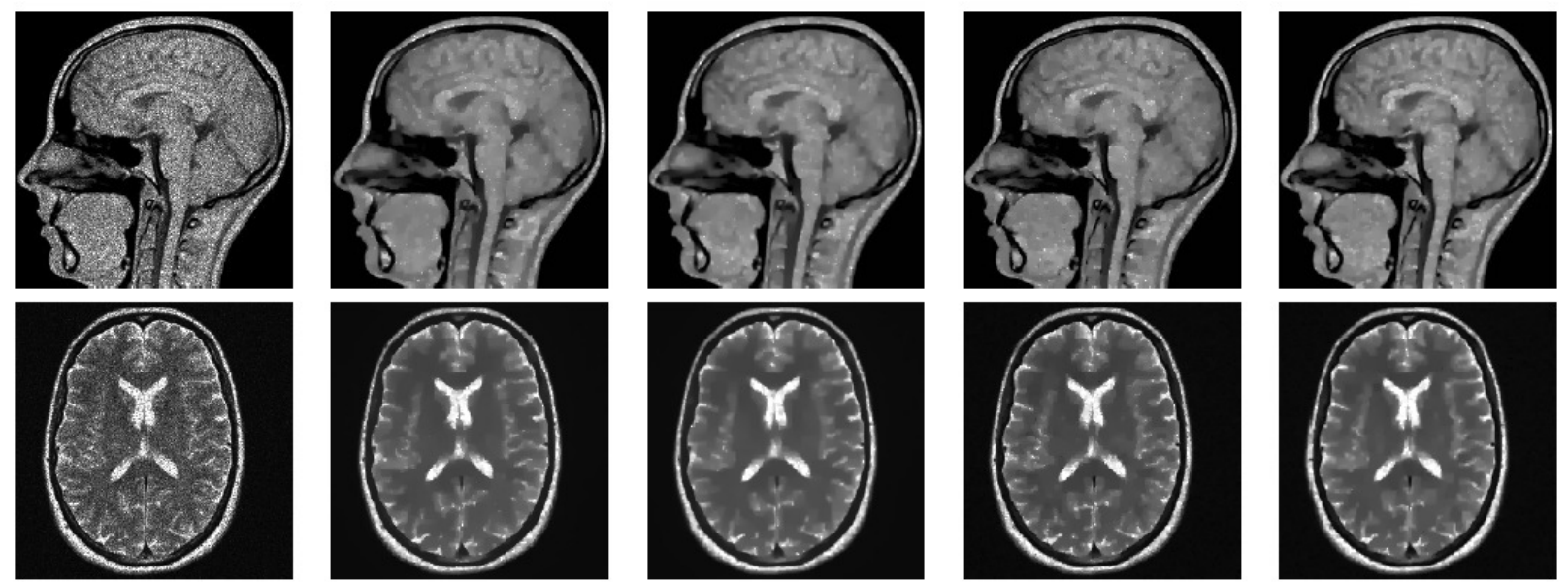



(a)

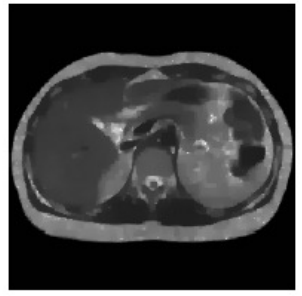

(b)

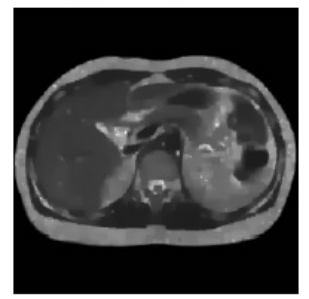

(c)

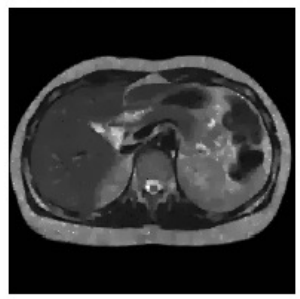

(d)

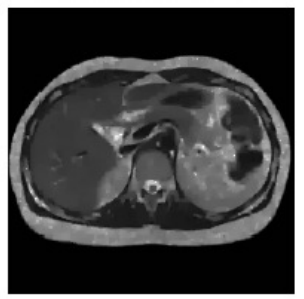

(e)

Figure 5. Recovered results for images. (a) Noisy image ( $L=10$ ), (b) M1 model, (c) M2 model, (d) M3 model, (e) Ours
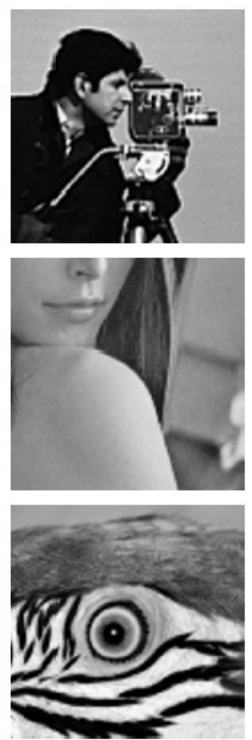

(a)
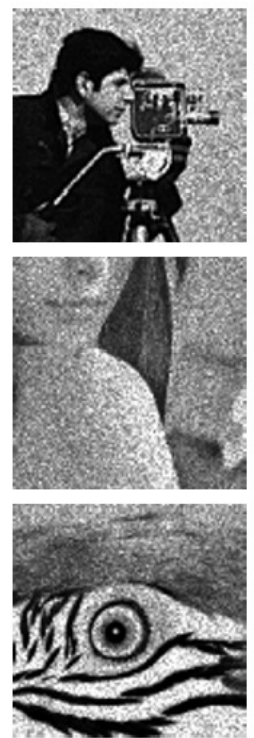

(b)
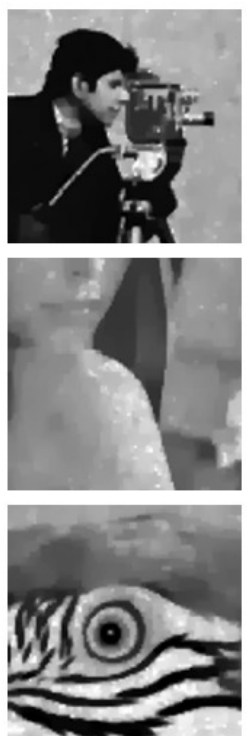

(c)
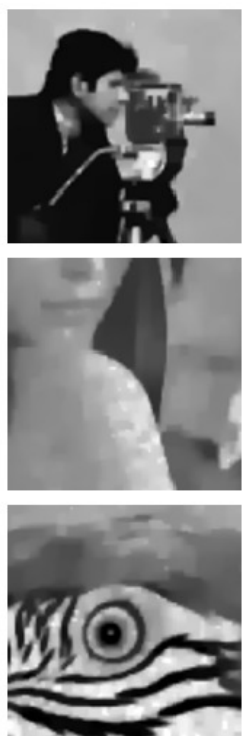

(d)
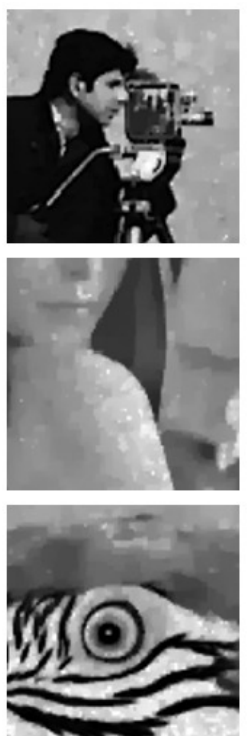

(e)
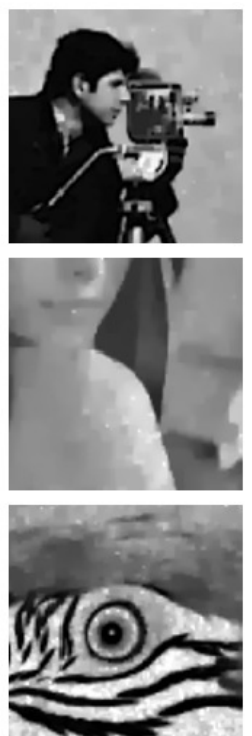

(f)

Figure 6. The zoomed-in part of the recovered images in Figure (2). (a) Details of original images; and details of restored images by: (b) M1 model, (c) M2 model, (d) M3 model, (e) ours

Chan, S.H., Khoshabeh, R., et al. (2011). An Augmented Lagrangian Method for Total Variation Video Restoration. IEEE Trans. Image Process., 20 (11), pp. 30973111.

Chambolle, A. (2004). An algorithm for total variation minimization and applications. J. Math. Imaging Vis., 20 pp. 89-97.
Chen, H. Z., Song, J. P., and Tai, X. C. (2009). A dual algorithm for minimization of the LLT model. $A d v$. Comput. Math., 31 pp. 115-130.

Chen, Y.M., and Wunderli, T. (2002). Adaptive total variation for image restoration in BV space. J. Math. Anal. Appl., 272 (1), pp. 117-137.

Chyba, M., and Marriott, J. (2012). Exceptional trajec- 

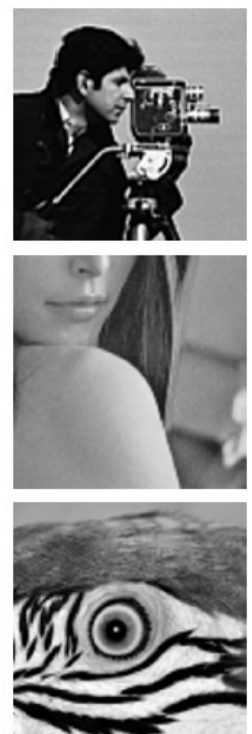

(a)
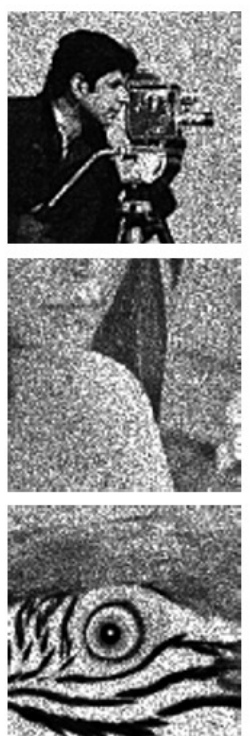

(b)
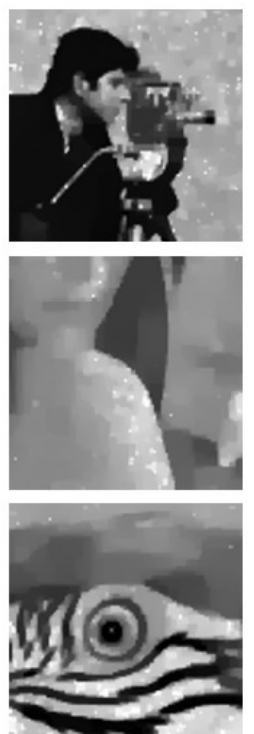

(c)
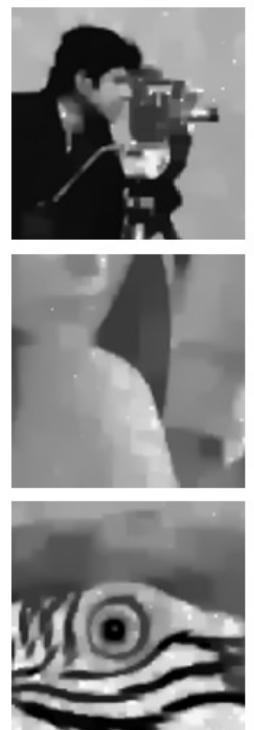

(d)
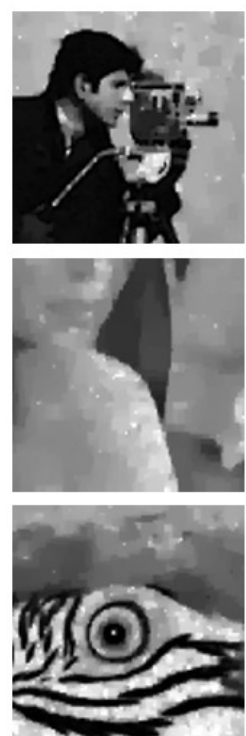

(e)
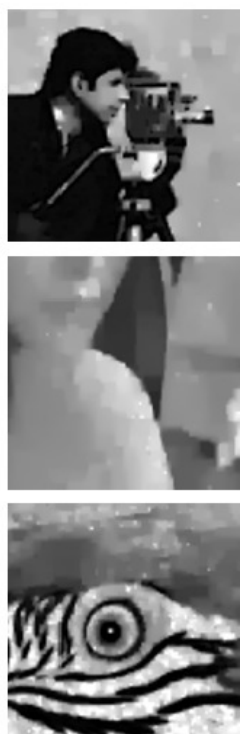

(f)

Figure 7. The zoomed-in part of the recovered images in Figure (3). (a) Details of original images; and details of restored images by: (b) M1 model, (c) M2 model, (d) M3 model, (e) ours
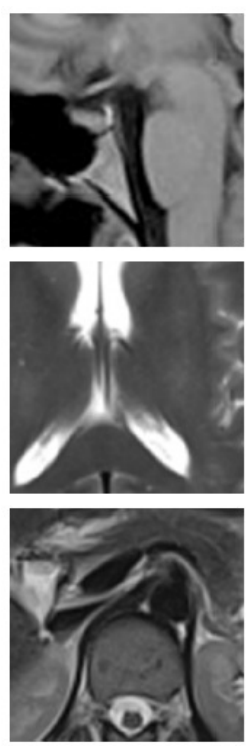

(a)
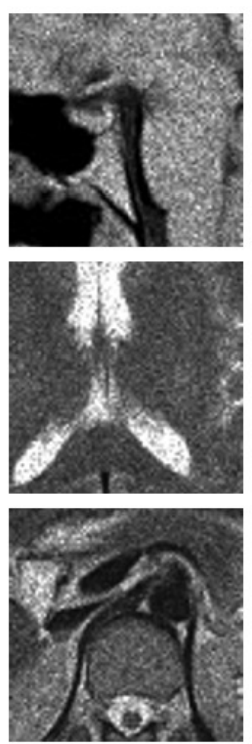

(b)
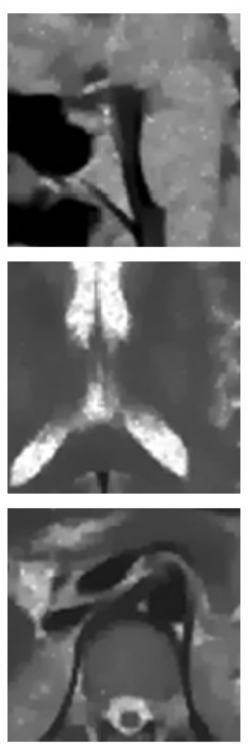

(c)
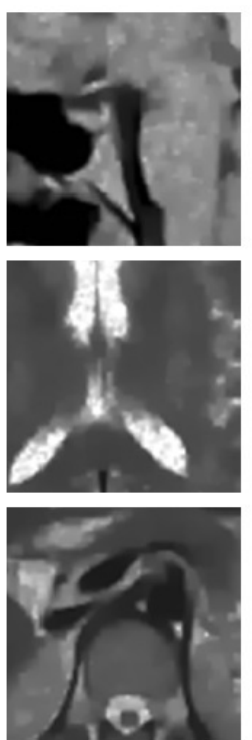

(d)
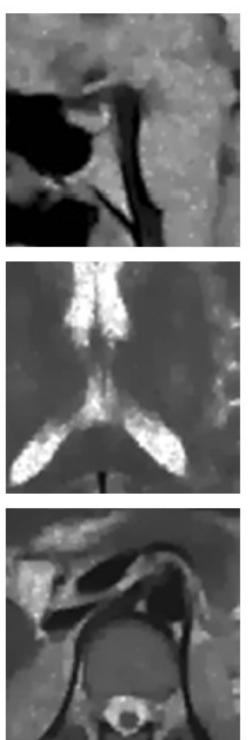

(e)
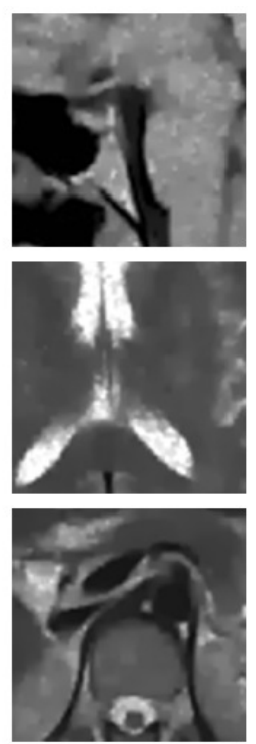

(f)

Figure 8. The zoomed-in part of the recovered images in Figure (4). (a) Details of original images; and details of restored images by: (b) M1 model, (c) M2 model, (d) M3 model, (e) ours

tories in the contrast problem in nuclear magnetic resonance. Cybernetics and Physics, 1 (4), pp. 243--251. Dong, J., Han, Z., Zhao, Y., Wang, W., et al. (2017). Sparse analysis model based multiplicative noise removal with enhanced regularization. Signal Process., 137 pp. $160-176$.

Dong, Y., and Zeng, T. (2013). A convex variational model for restoring blurred images with multiplicative noise. SIAM J. Imaging Sci., 6 (3), pp. 1598-1625.
Feinberg, E. A., Kasyanov, P. O., and Zadoianchuk, N. V. (2014). Fatou's Lemma for Weakly Converging Probabilities. Theory Probab. its Appl., 58 (4), pp. 683-689. Goldstein, T. and Osher, S. (2008). The split Bregman method for L1-regularized problems. SIAM J. Imaging Sci., 2 (2), pp. 323-343.

Goodman, Joseph W. (2020). Speckle Phenomena in Optics: Theory and Applications, Second Edition. Society of Photo-Optical Instrumentation Engineers, 468 p. 

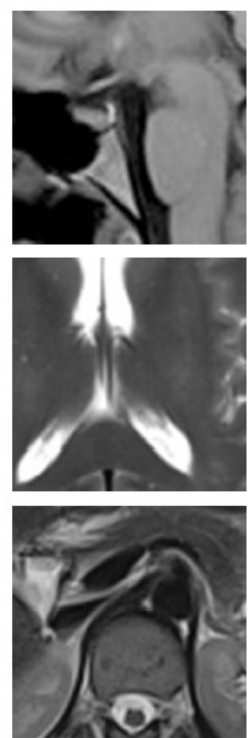

(a)
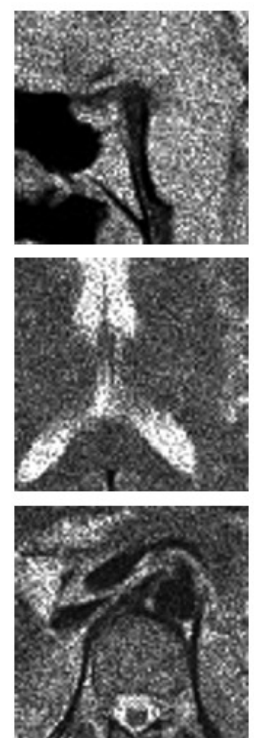

(b)
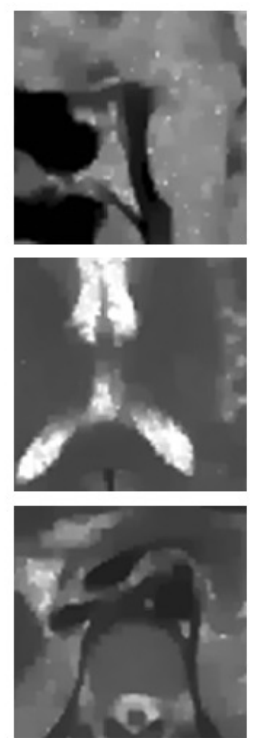

(c)
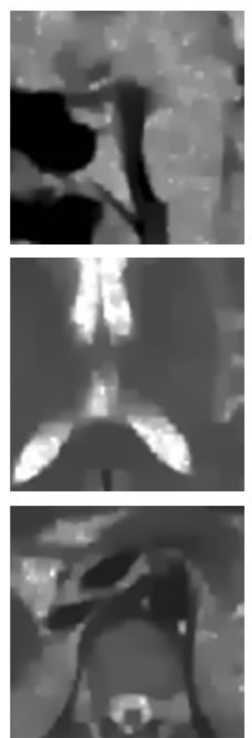

(d)
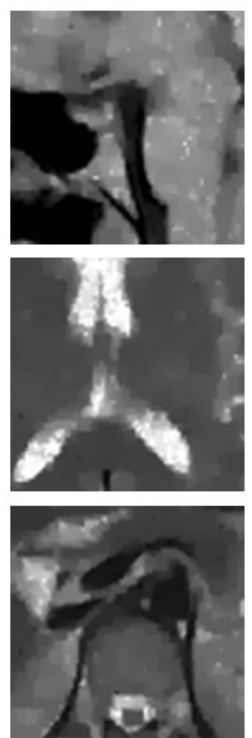

(e)
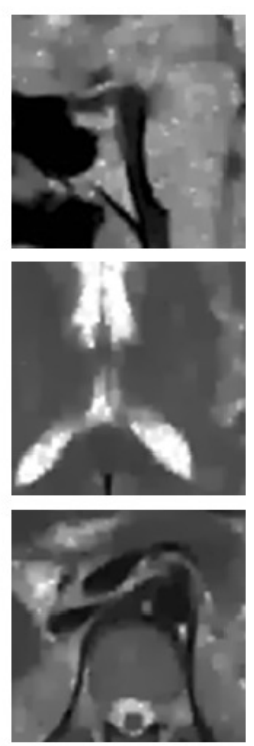

(f)

Figure 9. The zoomed-in part of the recovered images in Figure (5). (a) Details of original images; and details of restored images by: (b) M1 model, (c) M2 model, (d) M3 model, (e) ours

Granichin, O., Erofeeva, V., and Senin, I. (2018). Modifying the physical process of ultrasound tomography scanning through compressive sensing. Cybernetics and Physics, 7 (2), pp. 66-71.

Granwehr, J. (2007) Multiplicative or $t_{1}$ noise in NMR spectroscopy. Appl. Magn. Reson., 32 (2), pp. 113156.

Huang, Y.-M., Ng, M.K., and Wen, Y.W. (2009). A new total variation method for multiplicative noise removal. SIAM J. Imaging Sci., 2 (1), pp. 20-40.

Jiang, L., Huang, J., Liu, J., and Lv, X. (2014) A combined first-order and second-order variation approach for multiplicative noise removal. ANZIAM J., 56 (2), pp. 116-137.

Jin, Z., and Yang, X. (2011). A variational model to remove the multiplicative noise in ultrasound images. J. Math. Imaging Vis., 39 pp. 62-74.

Lefkimmiatis, S., Bourquard, A., and Unser M. (2012). Hessian-based norm regularization for image restoration with biomedical applications. IEEE Trans. Image Process., 21 (3), pp. 983-995.

Li, S., Wang, G., and Zhao, X. (2016). Multiplicative noise removal via adaptive learned dictionaries and TV regularization. Digit. Signal Process., 50 pp. 218-228

Li, Z., Lou, Y., and Zeng, T. (2016). Variational multiplicative noise removal by dc programming. J. Sci. Comput., 68 pp. 1200-1216.

Liu, J., Huang, T.Z., Xu, Z., and Lv, X.G.(2013). High-order total variation-based multiplicative noise removal with spatially adapted parameter selection. $J$. Opt. Soc. Am. A, 30 (10), pp. 1956-1966.

Liu, M., and Fan, Q. (2016) A modified convex variational model for multiplicative noise removal. $J$ Vis.
Commun. Image R., 36 pp. 187-198.

Liu, X., Zaki, F., and Renaud, D. (2018) Assessment and removal of additive noise in a complex optical coherence tomography signal based on Doppler variation analysis. Appl. Opt., 57 pp. 2873-2880.

Lysaker, M., and Tai, X. C. (2006). Iterative image restoration combining total variation minimization and a second-order functional. Int. J. Comput. Vis., 55 pp. 5-18.

Liu, Q., Yao, Z., and Ke, Y. (2007). Entropy solutions for a fourth-order nonlinear degenerate problem for noise removal. Nonlinear Anal. Theory Methods Appl., 67 (6), pp. 1908-1918.

Li, F., Shen, C., Fan, J., and Shen, C. (2007) Image restoration combining a total variational filter and a fourth-order filter. J Vis. Commun. Image R., 18 (4), pp. 322-330.

Lv, T., Han, X., Wu, S., and Li, Y. (2019). The effect of speckles noise on the Laser Doppler Vibrometry for remote speech detection. Opt. Commun., 440 (1), pp. 117-125.

Papafitsoros, K. and Schonlieb, C. B. (2014). A combined first and second order variational approach for image reconstruction. J. Math. Imaging Vis., 48 pp. 308-338.

Pham, C.T., Tran, T.T.T., and Gamard, G. (2020). An efficient total variation minimization method for image restoration. Informatica, 31 (3), pp. 539-560.

Pham, C.T., et al. (2019). An algorithm for image restoration with mixed noise using total variation regularization. Cybern. Phys., 8(2), pp. 73-82.

Pham, C.T., and Kopylov, A. V. (2018). Tree-serial parametric dynamic programming with flexible prior 
model for image denoising. Comput. Opt., 42(5), pp. 838-845.

Pham, C.T., Gamard, G., Kopylov, A., and Tran, T.T.T. (2018). An algorithm for image restoration with mixed noise using total variation regularization. Turk. J. Elec. Eng. \& Comp. Sci., 26 (6), pp. 2831-2845.

Pham, C.T., and Kopylov, A. (2015). Multi-quadratic dynamic programming procedure of edge-preserving denoising for medical images Int. Arch. Photogramm., XL-5-W6 pp. 101-106.

Rudin, L., Lions, P. L., and Osher, S. (2003). Multiplicative denoising and deblurring: theory and algorithms, in geometric level set methods in imaging vision, and graphics. Springer, New York, pp. 103-119.

Steidl, G. and Teuber, T. (2010). Removing multiplicative noise by Douglas-Rachford splitting methods. $J$. Math. Imaging Vis., 36 pp. 168-184.

Tabatabai, H., Oliver, D.E., Rohrbaugh, J.W., and Papadopoulos, C. Novel Applications of Laser Doppler Vibration Measurements to Medical Imaging. Sens. Imaging, 14 pp. 13-28.

Tai, X. C., Hahn, J., and Chung, G. J. (2011). A fast algorithm for Euler's elastica model using Augmented Lagrangian method. SIAM J. Imaging Sci., 4 (1), pp. 313-344.

Ullah, A., Chen, W., Khan, M. A., and Sun, H. (2017). A New Variational Approach for Multiplicative Noise and Blur Removal. PLoS ONE, 12 (1): e0161787, 26 p.

Vassa, J. et al. (2008). Avoidance of speckle noise in laser vibrometry by the use of kurtosis ratio: Application to mechanical fault diagnostics. Mech. Syst. Signal Process., 22 (3), pp. 647-671.

Yao, W., Guo, Z., Sun, J., et al. (2019). Multiplicative Noise Removal for Texture Images Based on Adaptive Anisotropic Fractional Diffusion Equations. SIAM J. Imaging Sci., 12 (2), pp. 839-873.

Zhao, X., Wang, F., and Ng, M. K. (2014). A new convex optimization model for multiplicative noise and blur removal, SIAM J. Imaging Sci., 7 (1), pp. 456-475.

Zhou, Z., Guo, Z., Dong, G., Sun, J., et al. (2015). A doubly degenerate diffusion model based on the gray level indicator for multiplicative noise removal. IEEE Trans. Image Process., 24 (1), pp. 249-260.

Zhu, J., Li, Y., and Baets, R. (2019). Mitigation of speckle noise in laser Doppler vibrometry by using a scanning average method. Opt. Lett., 44 (7), pp. 18601863.

Wu, C., and Tai, X.C. (2010). Augmented Lagrangian method, dual methods, and split Bregman iteration for ROF, vectorial TV, and high order models. SIAM J. Imaging Sci., 3 (3), pp. 300-339.

Wang, Y., Yang, J., Yin, W., and Zhang, Y. (2008). A New Alternating Minimization Algorithm for Total Variation Image Reconstruction. SIAM J. Imaging Sci., 1 (3), pp. 248-272.

Wang, Z. and Bovik, A.C. (2006). Modern Image Quality Assessment Synthesis Lectures on Image, Video, and Multimedia Processing. Morgan and Claypool Publishers, 2006, $156 \mathrm{p}$. 